\title{
DETERMINAÇÃO DO ZINCO E COBRE EM FERTILIZANTES POR ESPECTROFOTOMETRIA DE ABSORÇÃO ATÔMICA *
}

\author{
J. C. AlCARDE \\ C. O. PONCHIO
}

\section{RESUMO}

\begin{abstract}
O presente trabalho descreve os estudos efetuados sobre alguns aspectos dos métodos espectrofotométricos de absorção atômica, empregados na determinação do zinco e cobre em fertilizantes. Assim, foram objeto de estudos o efeito da acidez da solução da amostra, a influênca dos ânions fosfato, sulfato e nitrato, em função da acidez da solução da amostra, a durabilidade das soluções padrão e a precisão e a exatidão dos métodos.

Os resultados permitiram concluir que a absorbância das soluções padrão de zinco e cobre não é afetada pela concentração de $\mathrm{HCl}$, podendo esta variar de $0,1 \mathrm{~N}$ a $0,5 \mathrm{~N}$ e que os referidos métodos não sofrem a influência dos ânions fosfato, sulfato e nitrato, em qualquer concentração de $\mathrm{HC} 1$ dentro do intervalo entre $0,1 \mathrm{~N}$ e $0,5 \mathrm{~N}$. Finalmente, podese verificar que os métodos estudados são suficientemente precisos e exatos para a determinação do zinco e cobre em fertilizantes.
\end{abstract}

\section{INTRODUÇÃO}

O uso de fertilizantes contendo micronutrientes é, atualmente, uma prática que está se tornando comum entre nós, em virtude da carência desses elementos no solo e pelas vantagens que tal prática oferece.

A determinação de micronutrientes em fertilizantes é, em grande parte, feita por métodos gravimétricos e volumétricos, os quais são, em geral, morosos e de sensibilidade limitada (OFFICIAL METHODS OF ANALYSIS OF THE A.O.A.C., 1970). Alguns dos referidos elementos são determinados colorimetricamente.

Os métodos comumente empregados para a determinação do

* Entregue para publicação em 3-1-1973.

** Departamento de Química da E.S.A. "Luiz de Queiroz".

*** Aluno do 3.० ano da E.S.A. "Luiz de Queiroz". 
zinco em fertilizantes são: o gravimétrico, baseado na precipitação do zinco como $\mathrm{Zn}(\mathrm{OH})_{2}$ e o colorimétrico da ditizona (OFFICIAL METHODS OF ANALYSIS OF THE A.O.A.C., 1970). Por outro lado, o método mais utilizado para a determinação do cobre é o volumétrico, baseado na redução do $\mathrm{Cu}^{2+}$ a $\mathrm{Cu}^{1+}$ pelo iodeto de potássio, com consequente libertação de iodo, o qual é titulado com uma solução padronizada de $\mathrm{Na}_{2} \mathrm{~S}_{2} \mathrm{O}_{3}$ (OFFICIAL METHODS OF ANALYSIS OF THE A.O.A.C., 1970). Recentemente, ALCARDE, CATANI \& PONCHIO (1971) adaptaram o método colorimétrico do dietilditiocarbamato para a determinação do cobre em fertilizantes.

Métodos baseados na espectrofotometria de absorção atômica começaram a ser empregados na análise de fertilizantes por McBRIDE (1965). O referido autor apresentou um método onde, num mesmo extrato, são determinados magnésio, cobre, ferro, manganês e zinco. Este método, que passou a integrar a décima edição do OFFICIAL METHODS OF ANALYSIS OF THE A.O.A.C., (1965), já foi ampliado para a determinação do cálcio e também foi adaptado para a determinação dos citados elementos em fertilizantes orgânicos e "fritas" (McBRIDE, 1967 e 1968; ALLAN, 1969; OFFICIAL METHODS OF ANALYSIS OF THE A.O.A.C., 1970).

O objetivo do presente trabalho foi o de estudar alguns aspectos dos métodos espectrofotométricos de absorção atômica empregados na determinação do zinco e do cobre em fertilizantes. Foram objetos de estudos o efeito da acidez da solução da amostra, a influência dos ânions fosfato, sulfato e nitrato em função da acidez da solução da amostra, a durabilidade das soluções padrão e a precisão e a exatidão dos métodos.

\section{MATERIAL E MÉTODOS}

\section{Material}

O material constou de duas misturas de fertilizantes, caracterizadas como $M_{1}$ e $M_{2}$ preparadas em laboratório a partir de fertilizantes simples comerciais, e cuja composição está mostrada no quadro 1. O preparo dessas misturas foi efetuado colocando-se todos os componentes num gral onde se fez a trituração e homogeneização. 
QUADRO 1. - Composição das misturas de fertilizantes preparadas em laboratório.

\begin{tabular}{lll}
\hline & & Porcentagem de cada componente \\
\cline { 2 - 3 } Componentes & $\mathbf{M}_{1}$ & $\mathbf{M}_{2}$ \\
\hline Superfosfato simples & 30 & 20 \\
Superfosfato triplo & 20 & 10 \\
Cloreto de potássio & 20 & - \\
Salitre do Chile & 10 & 20 \\
Uréia & - & 20 \\
Sulfato de amônio & 20 & 30 \\
\hline
\end{tabular}

\section{Reativos}

Solução de HC1 $(1+1)$ destilado.

Solução de HC1 0,1 N, 0,25 N e 0,5 $N$ (destilado).

Solução padrão estoque de zinco, contendo 1000 microgramas de $\mathbf{Z n}^{2+}$ por mililitro - Transferir $1,000 \mathrm{~g}$ de zinco metálico, p.a., para copo de $150 \mathrm{ml}$, adicionar $10 \mathrm{ml}$ de solução de HC1 $(1+1)$, cobrir com vidro de relógio e ferver 2-3 minutos. Retirar o vidro de relógio, deixar evaporar até quase secar e diluir a 1 litro, com água desmineralizada.

Solução padrão de zinco contendo 100 microgramas de $\mathrm{Zn}^{2+}$ por mililitro (Solução A) - Diluir $25 \mathrm{ml}$ da solução estoque a 250 $\mathrm{ml}$ com solução $0,1 \mathrm{~N}$ ou $0,25 \mathrm{~N}$ ou $0,5 \mathrm{~N}$ de HC1.

Solução padrão de zinco contendo 25 microgramas de $\mathrm{Zn}^{2+}$ por mililitro (Solução B) - Diluir $25 \mathrm{ml}$ da solução A a $100 \mathrm{ml}$ com solução $0,1 \mathrm{~N}$ ou $0,25 \mathrm{~N}$ ou $0,5 \mathrm{~N}^{\prime}$ de $\mathrm{HC}$.

.Solução padrão de trabalho de zinco, contendo 0,5 - 1,0 1,5 - 2,0 - 2,5 e 3,0 microgramas de $\mathrm{Zn}^{2+}$ por mililitro - Trans ferir 2, 4, 6, 8, 10 e 12 mililitros da solução $\mathbf{B}$ para balóes de $100 \mathrm{ml}$ e completar o volume com solução de $\mathrm{HC} 10-1 \mathrm{~N}$ ou $0,25 \mathrm{~N}$ ou $0,5 \mathrm{~N}$.

Solução padrão estoque de cobre, contendo 1000 microgramas de $\mathrm{Cu}^{2+}$ por mililitro - Transferir 1,0000 de cobre eletrolítico, p.a., para copo de $150 \mathrm{ml}$, adicionar $10 \mathrm{ml}$ de solução de HC1 $(1+1)$ e $2 \mathrm{ml}$ de solução de $\mathrm{HNO}_{3}$ concentrado. Cobrir com vidro de relógio 
e ferver por 2-3 minutos. Retirar o vidro de relógio, deixar evaporar até quase secar e diluir a 1 litro com água desmineralizada.

Solução padrão de cobre contendo 100 microgramas de $\mathrm{Cu}^{2+}$ por mililitro (Solução C) - Diluir $25 \mathrm{ml}$ da solução estoque a $250 \mathrm{ml}$ com solução $0,1 \mathrm{~N}$ ou $0,25 \mathrm{~N}$ ou $0,5 \mathrm{~N}$ de $\mathrm{HC}$.

Soluções padrão de trabalho de cobre contendo $2,0-4,0-$ 8,0 - 12,0 - 16,0 e 20,0 microgramas de $\mathrm{Cu}^{2+}$ por mililitro Transferir $2,4,8,12,16$ e $20 \mathrm{ml}$ da solução $\mathbf{C}$ para balões volumétricos de $100 \mathrm{ml}$ e completar o volume com solução $0,1 \mathrm{~N}$ ou $0,25 \mathrm{~N}$ ou $0,5 \mathrm{~N}$ de $\mathrm{HC} 1$.

\section{Métodos}

\section{1 - Preparo dos extratos de fertilizantes}

\section{1 - Em solução 0,1 $\mathbf{N}$ de HC1}

a) Pesar 1,000 g da amostra finamente moída, transferir para copo de $150 \mathrm{ml}$ e acrescentar $20 \mathrm{ml}$ de solução de HC1 (1+1).

b) Ferver lentamente e deixar evaporar até quase secar (não deixar calcinar).

c) Retomar o resíduo com $20 \mathrm{ml}$ de solução $0,5 \mathrm{~N}$ de $\mathrm{HCl} \mathrm{e}$ filtrar por papel Watman $n .^{\circ} 1$, recebendo o filtrado num balão volumétrico de $100 \mathrm{ml}$. Lavar o copo e o filtro com 5 porções de $10 \mathrm{ml}$ de água desmineralizada, completar o volume e agitar.

d) Desenvolver uma prova em branco.

\section{2 - Em solução $0,5 \mathrm{~N}$ de $\mathrm{HC1}$}

Proceder conforme descrito em 1.1, apenas que no item c deve-se retomar o resíduo com $20 \mathrm{ml}$ de solção $2 \mathrm{~N}$ de $\mathrm{HC1}$.

\section{2 - Determinação do zinco}

a) Acertar o espectrofotômetro de absorção atômica nas condições ótimas para a determinação do zinco (foi utilizado o aparelho Perkin-Elmer, Modelo 303, nas condições descritas no quadro 2).

b) Ler a absorção dos extratos de fertilizantes, contra a prova em branco (solução de HC1 $0,1 \mathrm{~N}$ ou $0,5 \mathrm{~N}$ ), diretamente ou diluir com solução de $\mathrm{HC} 10,1 \mathrm{~N}$ ou $0,5 \mathrm{~N}$, conforme o caso, para obter soluções com uma concentração de zinco dentro do intervalo usado nas soluções padrão de trabalho.

c) Ler a absorção das soluções padrão de trabalho de zinco 
no início e após a leitura de grupos de, no máximo, 6 amostras. Com a média das obsorbancias, relacioná-las com as concentrações de zinco e confeccionar a curva padrão.

\section{3 - Determinação do cobre}

a) Acertar o espectrofotômetro de absorção atômica nas condições ótimas para a determinação do cobre (foi utilizado o aparelho Perkin-Elmer, Modelo 303, nas condições descritas no quadro 2).

b) Prosseguir conforme descrito a partir do item $\mathbf{b}$ da determinação do zinco.

QUADRO 2 - Condições instrumentais usadas no espectrofotômetro de absorção atômica Perkin-Elmer, Modelo 303, para a determinação do zinco e do cobre em fertilizantes.

\begin{tabular}{lcc}
\hline & \multicolumn{2}{c}{ Condições usadas } \\
\cline { 2 - 3 } & Zariáveis & Cobre \\
\hline Comprimento da onda (angstrons) & 2138 & 3247 \\
Largura do "slit" & n..$^{\circ}$ & n. ${ }^{\circ} 4$ \\
Fonte (m.a.) & 15 & 10 \\
Ar & 4,5 & 4,5 \\
Acetileno & 9 & 9 \\
Chama & oxidante & oxidante \\
Altura do bico & $1 / 8$ & $1 / 8$ \\
Intervalo de concentração & 0,5 a 3,0 ppm & 2,0 a 20,0 ppm \\
\hline
\end{tabular}

\section{RESULTADOS OBTIDOS E DISCUSSÃO}

\section{Durabilidade das soluções padrão de trabalho}

Constitui recomendação comum que os padrões de trabalho devem ser preparados diariamente (McBRIDE, 1965 e 1967; OFFICIAL METHODS OF ANALYSIS OF THE A.O.A.C., 1970).

O presente estudo foi desenvolvido com o objetivo de verificar a conservação das soluções padrão de trabalho. Tais soluções, pre- 
paradas em solução $0,5 \mathrm{~N}$ de $\mathrm{HC} 1$, foram mantidas à temperatura ambiente durante 6 meses e a absorbância das mesmas foi determinada periodicamente. Os resultados obtidos para as soluções padrão de zinco estão descritos no quadro 3 e para as soluções padrão de cobre no quadro 4.

QUADRO 3. - Absorbâncias das soluções padrão de zinco, preparadas em solução de $\mathrm{HC} 10,5 \mathrm{~N}$, após diversos períodos de tempo.

\begin{tabular}{cccc}
\hline \multirow{2}{*}{$\begin{array}{c}\text { Padrões de zinco } \\
(\mathrm{ppm})\end{array}$} & \multicolumn{3}{c}{ Tempo de conservação (meses) } \\
\cline { 2 - 4 } & 0 & 2 & 6 \\
\hline 0,5 & 0,07 & 0,07 & 0,06 \\
1,0 & 0,12 & 0,12 & 0,12 \\
2,0 & 0,24 & 0,24 & 0,24 \\
3,0 & 0,38 & 0,38 & 0,37 \\
\hline
\end{tabular}

QUADRO 4. - Absorbâncias das soluções padrão de cobre, preparadas em solução de $\mathrm{HC1}$ 0,5 N, após diversos períodos de tempo.

\begin{tabular}{cccc}
\hline \multirow{2}{*}{$\begin{array}{c}\text { Padrões de cobre } \\
(\mathrm{ppm})\end{array}$} & 0 & 2 & 6 \\
\cline { 2 - 4 } & 0,09 & 0,09 & 0,09 \\
4,0 & 0,26 & 0,27 & 0,26 \\
12,0 & 0,35 & 0,35 & 0,34 \\
16,0 & & & \\
\hline
\end{tabular}

Estes resultados demonstram que as soluções padrão de trabalho de zinco e cobre, preparadas em solução $0,5 \mathrm{~N}$ de HC1, conservam-se bem, durante 6 meses, à temperatura ambiente.

\section{Influência da acidez da amostra}

As determinações do zinco e cobre em fertilizantes por absorção atômica foram inicialmente apresentadas com as soluções padrão e os extratos preparados em solução 0,1 N de HC1 (McBRIDE, 1965). 
Posteriormente, esses métodos foram descritos empregando as soluções padrão e os extratos preparados em solução $0,5 \mathrm{~N}$ de HC1 (McBRIDE, 1967 e 1968; OFFICIAL METHODS OF ANALYSIS OF THE A.O.A.C., 1970).

Para verificar a influência da acidez sobre a absorbância das soluções, os padrões de zinco e cobre foram preparados em soluções $0,1 \mathrm{~N}-0,25$ e $0,50 \mathrm{~N}$ de $\mathrm{HCl}$ e suas absorbâncias foram determinadas. Os resultados da influência da acidez sobre a absorbância das soluções padrão de zinco estão apresentados no quadro 5 e a influência da acidez sobre as absorbâncias das soluções padrão de cobre no quadro 6.

QUADRO 5. - Absorbâncias das soluções padrão de zinco, preparadas em soluções de HC1 de diferentes concentrações.

\begin{tabular}{cccc}
\hline \multirow{2}{*}{$\begin{array}{c}\text { Padrões de zinco } \\
(\mathrm{ppm})\end{array}$} & \multicolumn{4}{c}{ Concentração de $\mathrm{HC1}$ nas soluções padrão } \\
\cline { 2 - 4 } & $0,1 \mathrm{~N}$ & $0,25 \mathrm{~N}$ & $0,50 \mathrm{~N}$ \\
\hline 1,0 & 0,16 & 0,16 & 0,16 \\
3,0 & 0,49 & 0,48 & 0,49 \\
\hline
\end{tabular}

QUADRO 6. - Absorbâncias das soluções padrão de cobre, preparadas em soluções de HC1 de diferentes concentrações.

\begin{tabular}{cccc}
\hline \multirow{2}{*}{$\begin{array}{c}\text { Padrões de cobre } \\
(\mathrm{ppm})\end{array}$} & \multicolumn{3}{c}{ Concentração de $\mathrm{HC1}$ nas soluções padrão } \\
\cline { 2 - 4 } & $0,1 \mathrm{~N}$ & $0,25 \mathrm{~N}$ & $0,50 \mathrm{~N}$ \\
\hline 4,0 & 0,10 & 0,11 & 0,11 \\
16,0 & 0,41 & 0,40 & 0,40 \\
\hline
\end{tabular}

Os resultados estão mostrando que a absorbância das soluções padrão de zinco e cobre não sofre influência da concentração de $\mathrm{HC}$, dentro do intervalo compreendido entre $0,1 \mathrm{~N}$ e $0,5 \mathrm{~N}$.

Influência dos ânions fosfato, sulfato e nitrato, em função da concentração de HC1 na solução

As misturas de fertilizantes podem conter os ânions fosfato, 
sulfato e nitrato em elevada concentração. Por este motivo, e em virtude dos dados apresentados por SLAVIN (1968) citando diversos autores, a possibilidade da influência destes ânions nos métodos em apreço foi estudada, levando em conta a concentração de HC1 presente na solução.

Foram preparadas soluções padrão de zinco e de cobre, em soluções $0,1 \mathrm{~N}-0,25 \mathrm{~N}$ e $0,5 \mathrm{~N}$ de $\mathrm{HC}$, e contendo, isoladamente, as seguintes concentrações dos íons cuja interferência deveria ser estudada: $0-1-2$ e $3 \mathrm{mg}$ de $\mathrm{PO}_{4}^{3-}\left(\mathrm{K}_{2} \mathrm{HPO}_{4}\right)$ por mililitro; $0-$ $1-2$ e $3 \mathrm{mg}$ de $\mathrm{SO}_{4}^{2-}\left(\mathrm{K}_{2} \mathrm{SO}_{4}\right)$ por mililitro; $0-2,5-5$ e $7,5 \mathrm{mg}$ de $\mathrm{NO}_{3}\left(\mathrm{KNO}_{3}\right)$ por mililitro. Os resultados relativos às soluções padrão de zinco acham-se descritos no quadro $7 \mathrm{e}$ os relativos às soluções padrão de cobre no quadro 8 .

Observando-se os dados dos quadros 7 e 8 nota-se que a absorbância das soluções padrão de zinco e de cobre, preparadas em soluções cuja concentração de $\mathrm{HCl}$ variou de 0,1 a $0,5 \mathrm{~N}$, não sofreram a influência dos ânions fosfato, sulfato e nitrato, dentro das concentrações estudadas. Como uma única exceção, parece que a absorbância das soluções padrão de zinco, preparadas em soluções cuja concentração de $\mathrm{HC} 1$ foi $0,5 \mathrm{~N}$, sofreram ligeira influência do ânion nitrato quando a concentração desta foi de $7,5 \mathrm{mg} / \mathrm{ml}$.

\section{Estudo da precisão e da exatidão}

O estudo da precisão e da exatidão dos métodos foi feito através de um ensaio de recuperação, com 5 repetições. Às misturas $M_{1}$ e $M_{2}$ foram adicionadas quantidades variáveis de zinco e cobre, através de soluções padrão destes elementos, após a pesagem das amostras Em seguida, o conteúdo de zinco e cobre foi determinado em extratos preparados com solução $0,1 \mathrm{~N}$ e $0,5 \mathrm{~N}$ de $\mathrm{HC1}$. Os resultados acham-se descritos nos quadros 9 e 10.

Os dados dos quadros 9 e 10 estão demonstrando que os métodos espectrofotométricos de absorção atômica de determinação do zinco e do cobre em fertilizantes são adequadamente precisos e exatos. Alguns valores como $9,45 \%-7,85 \%-6,28 \%$ para o coeficiente de variação e $108,5 \%$ e $96,0 \%$ para a recuperação podem parecer, à primeira vista, insatisfatórios; mas, considerando a concentração relativamente baixa de zinco e cobre que deve existir no extrato de fertilizantes a ser submetido à leitura da absorção, tais valores são aceitáveis. É possível notar também que não houve diferença apreciável entre os resultados obtidos com os extratos preparados em solução de $\mathrm{HC} 10,1 \mathrm{~N}$ e $0,5 \mathrm{~N}$. 
QUADRO 7. - Absorbâncias das soluções padrão de zinco, preparadas com diversas concentrações de $\mathrm{HCl}$ e na presença de diferentes concentrações de fosfato, sulfato e nitrato.

\begin{tabular}{|c|c|c|c|c|c|c|}
\hline \multirow{3}{*}{$\begin{array}{l}\text { Concentração } \\
\text { do interferente } \\
\text { nos padrões } \\
(\mathrm{mg} / \mathrm{ml})\end{array}$} & \multirow{2}{*}{\multicolumn{3}{|c|}{$\frac{\text { Padrão de } \mathrm{Zn}^{2}+: 1,0 \mathrm{ppm}}{\text { Conc. de } \mathrm{HC} 1 \text { no padrão }}$}} & \multirow{2}{*}{\multicolumn{3}{|c|}{$\frac{\text { Padrão de } \mathrm{Zn}^{2+}: 3,0 \mathrm{ppm}}{\text { Conc. de } \mathrm{HC} 1 \text { no padrão }}$}} \\
\hline & & & & & & \\
\hline & $0,1 \mathrm{~N}$ & $0,25 \mathrm{~N}$ & $0,5 \mathrm{~N}$ & $0,1 \mathrm{~N}$ & $0,25 \mathrm{~N}$ & $0,5 \mathrm{~N}$ \\
\hline \multicolumn{7}{|l|}{$\mathrm{PO}_{4}^{3-}$} \\
\hline 0 & 0,14 & 0,15 & 0,14 & 0,43 & 0,43 & 0,43 \\
\hline 1 & 0,15 & 0,14 & 0,14 & 0,43 & 0,43 & 0,43 \\
\hline 2 & 0,14 & 0,15 & 0,14 & 0,43 & 0,43 & 0,43 \\
\hline 3 & 0,13 & 0,15 & 0,14 & 0,43 & 0,43 & 0,43 \\
\hline \multicolumn{7}{|l|}{$\mathrm{SO}_{4}^{2-}$} \\
\hline 0 & 0,16 & 0,16 & 0,16 & 0,49 & 0,48 & 0,49 \\
\hline 1 & 0,16 & 0,16 & 0,16 & 0,49 & 0,48 & 0,48 \\
\hline 2 & 0,16 & 0,15 & 0,16 & 0,48 & 0,48 & 0,49 \\
\hline 3 & 0,16 & 0,15 & 0,16 & 0,48 & 0,48 & 0,48 \\
\hline \multicolumn{7}{|l|}{$\mathrm{NO}_{3}^{-}$} \\
\hline 0,0 & 0,15 & 0,15 & 0,15 & 0,48 & 0,47 & 0,47 \\
\hline 2,5 & 0,15 & 0,15 & 0,15 & 0,48 & 0,47 & 0,47 \\
\hline 5,0 & 0,15 & 0,16 & 0,15 & 0,47 & 0,46 & 0,46 \\
\hline 7,5 & 0,15 & 0,15 & 0,14 & 0,48 & 0,47 & 0,44 \\
\hline
\end{tabular}


QUADRO 8. - Absorbâncias das soluções padrão de cobre, preparadas com diversas concentrações de $\mathrm{HC} 1$ e na presença de diferentes concentrações de fosfato, sulfato e nitrato.

\begin{tabular}{|c|c|c|c|c|c|c|}
\hline \multirow{3}{*}{$\begin{array}{l}\text { Concentração } \\
\text { do interferente } \\
\text { nos padrões } \\
(\mathrm{mg} / \mathrm{ml})\end{array}$} & \multirow{2}{*}{\multicolumn{3}{|c|}{$\frac{\text { Padrão de } \mathrm{Cu}^{2+}: 4,0 \mathrm{ppm}}{\text { Conc. de } \mathrm{HC} 1 \text { no padrão }}$}} & \multirow{2}{*}{\multicolumn{3}{|c|}{$\frac{\text { Padrão de } \mathrm{Cu}^{2+}: 16,0 \mathrm{ppm}}{\text { Conc. de } \mathrm{HC} 1 \text { no padrão }}$}} \\
\hline & & & & & & \\
\hline & $0,1 \mathrm{~N}$ & $0,25 \mathrm{~N}$ & $0,5 \mathrm{~N}$ & $0,1 \mathrm{~N}$ & $0,25 \mathrm{~N}$ & $0,5 \mathrm{~N}$ \\
\hline \multicolumn{7}{|l|}{$\mathrm{PO}_{4}^{3-}$} \\
\hline 0 & 0,10 & 0,11 & 0,11 & 0,41 & 0,40 & 0,40 \\
\hline 1 & 0,10 & 0,11 & 0,11 & 0,40 & 0,40 & 0,40 \\
\hline 2 & 0,10 & 0,11 & 0,11 & 0,40 & 0,40 & 0,40 \\
\hline 3 & 0,10 & 0,11 & 0,11 & 0,40 & 0,40 & 0,40 \\
\hline \multicolumn{7}{|l|}{$\mathrm{SO}_{4}^{2-}$} \\
\hline 0 & 0,10 & 0,11 & 0,10 & 0,40 & 0,38 & 0,39 \\
\hline 1 & 0,10 & 0,11 & 0,10 & 0,41 & 0,38 & 0,39 \\
\hline 2 & 0,10 & 0,11 & 0,10 & 0,41 & 0,37 & 0,39 \\
\hline 3 & 0,10 & 0,11 & 0,10 & 0,38 & 0,39 & 0,39 \\
\hline \multicolumn{7}{|l|}{$\mathrm{NO}_{3}$} \\
\hline 0,0 & 0,10 & 0,10 & 0,10 & 0,38 & 0,38 & 0,38 \\
\hline 2,5 & 0,10 & 0,10 & 0,10 & 0,38 & 0,38 & 0,39 \\
\hline 5,0 & 0,10 & 0,10 & 0,10 & 0,38 & 0,38 & 0,39 \\
\hline 7,5 & 0,10 & 0,10 & 0,10 & 0,38 & 0,38 & 0,39 \\
\hline
\end{tabular}




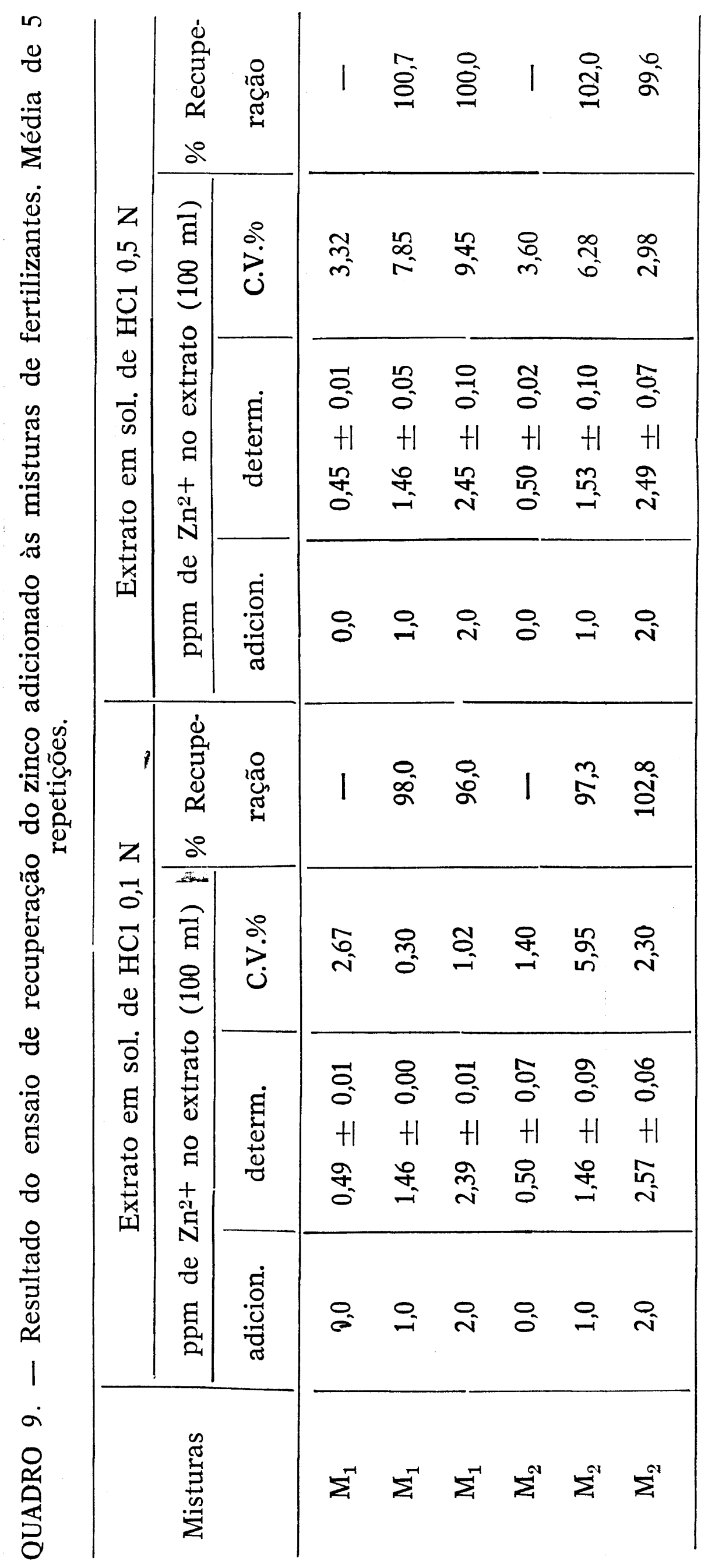




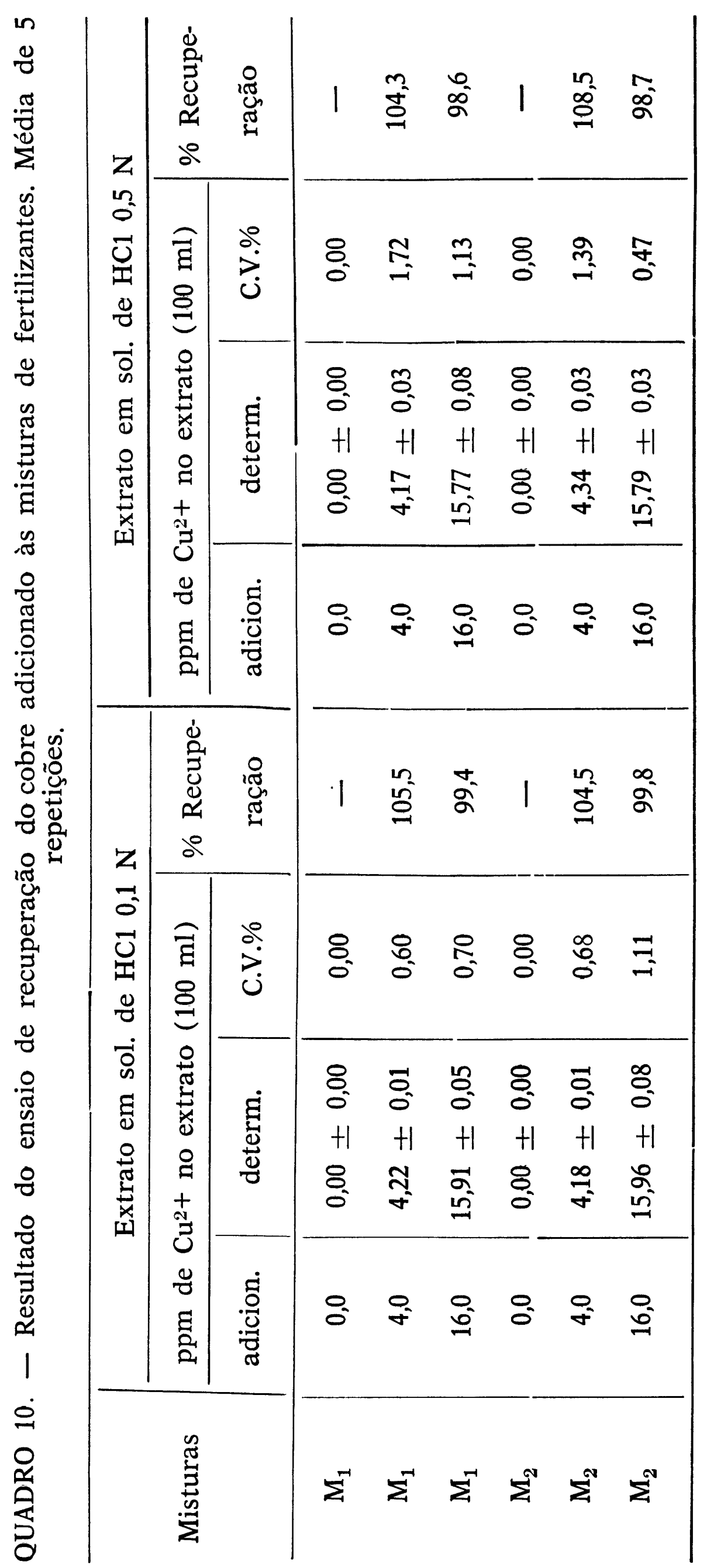




\section{CONCLUSÕES}

Os resultados obtidos no presente trabalho, permitiram concluir que :

a) a absorbância das soluções padrão de zinco e cobre não é afetada pela concentração de $\mathrm{HC1}$, podendo esta variar de $0,1 \mathrm{~N}$ a $0,5 \mathrm{~N}$.

b) os métodos em apreço não sofrem interferência dos ânions fosfato, sulfato e nitrato, em qualquer concentração de HC1 dentro do intervalo entre $0,1 \mathrm{~N}$ e $0,5 \mathrm{~N}$.

c) finalmente, os métodos estudados são adequadamente precisos e exatos para a determinação do zinco e cobre em fertilizantes, não havendo diferença nessas características em função da concentração de $\mathrm{HC1}$ no extrato de fertilizante.

\section{SUMMARY}

\section{DETERMINATION OF ZINC AND COPPER IN FERTILIZERS BY ATOMIC ABSORPTION SPECTROPHOTOMETRY.}

The determinations of zinc and copper in fertilizers by atomic absorption spectrophotometry are not influenced by the concentration of $\mathrm{HCl}$ in the extracts, in the range of $0,1 \mathrm{~N}$ to $0,5 \mathrm{~N}$ and neither by the anions phosphate, sulfate and nitrate in the same range of $\mathrm{HCl}$ concentration. The methods are precise and accurate for the determinations of zinc and copper in fertilizers.

\section{LITERATURA CITADA}

ALCARDE, J. C., R. A. CATANI \& C. O. PONCHIO, 1971 - Determinação colorimétrica do cobre em fertilizantes. Anais da ESALQ, 28:247-255.

ALLAN, J. E., 1969 - The preparation of agricultural samples for analysis by atomic absorption spectrophotometry. Variantechtron, $15 \mathrm{pp}$.

McBRIDE, C. H., 1965 - Determination of micronutrients in fertilizers by atomic absorption spectrophotometry. J. Ass. Off. Anal. Chem. 48(2): 406-413.

MCBRIDE, C. H., 1967 - Determination of secondary and minor plant nutrients in third collaborative study. J. Ass. Off. Anal. Chem., 50(2): 401-407.

MCBRIDE, C. H., 1968 - Determination of secondary and minor plant nutrients in fertilizers by atomic absorption spectrophotometry. J. Ass. Off. Anal. Chem., 51(4):847-851. 
OFFICIAL METHODS OF ANALYSIS OF THE A.O.A.C., 1965 - 10th ed., p. 23-24. Published by the Ass. of Off. Anal. Chem., Washington, D.C.

OFFICIAL METHODS OF ANALYSIS OF THE A.O.A.C., 1970 - 11th ed., p. 23-24. Published by Ass. of Off. Anal. Chem., Washington, D.C.

SLAVIN, W., 1968 - Atomic Absorption Spectrophotometry. Interscience Publishers. New York. $307 \mathrm{pp}$. 